\title{
Expression Profile of Osteoclasts Following the Stimulation With Interleukin-23 in Mice
}

\author{
Miao CHEN ${ }^{1}\left(\mathbb{D}\right.$, Dan-Dan PANG $^{2}$ (I), Sheng-Ming DAI ${ }^{1}$ (D) \\ ${ }^{1}$ Department of Rheumatology \& Immunology, Shanghai Jiao Tong University Affiliated Sixth People's Hospital, Shanghai, China \\ ${ }^{2}$ Department of Rheumatology \& Immunology, Changhai Hospital, Second Military Medical University, Changhai Hospital, \\ Second Military Medical University, Shanghai, China
}

\begin{abstract}
Objectives: This study aims to analyze the expression profile of osteoclasts (OCs) following the stimulation with interleukin 23 (IL-23) in mice, which would imply the underlying effects of IL-23 on the function of OCs in inflammatory arthritis.

Materials and methods: Mature OCs were induced from bone marrow mononuclear cells of 5 male mice (age 6 weeks; weighing $18-20 \mathrm{~g}$ ) in the presence of macrophage-colony stimulating factor $(50 \mathrm{ng} / \mathrm{mL})$ and receptor activator of nuclear factor kappa B ligand (30 ng/mL) in vitro. The Agilent SurePrint G3 Mouse GE V2.0 Microarray was used to analyze the gene expression profile of OCs stimulated with IL-23 ( 30 ng/mL) or vehicle. The four major IL-23-modulated genes were validated by quantitative real-time polymerase chain reaction (qPCR) analysis.

Results: The expression levels of 23 genes were up-regulated and 32 genes were down-regulated by IL-23 stimulation (fold change $\geq 1.5$ and $p$ value $<0.05)$. Among them, there were 37 genes with assigned gene symbols. Gene ontology analysis showed that the IL-23-regulated messenger ribonucleic acids (mRNAs) were related to positive regulation of leukocyte chemotaxis, chemokine-mediated signaling pathway and $\mathrm{C}-\mathrm{X}-\mathrm{C}$ chemokine receptors binding. The pathway analysis showed that the IL-23-regulated mRNAs were related to chemokine signaling pathway and cytokine-cytokine receptor interaction. The significant up-regulation of chemokine (C-X-C motif) ligand 1 and chemokine (C-X-C motif) ligand 2 induced by IL-23 was confirmed by qPCR. In addition, there were 18 long non-coding RNAs that were regulated by IL-23, while their function needs to be confirmed in the future.

Conclusion: Expression levels of genes related to chemotaxis in OCs were up-regulated by IL-23 in mice, which imply that IL-23 may facilitate chemotaxis of OCs in inflammatory arthritis.

Keywords: Chemokine (C-X-C motif) ligand 1, chemokine (C-X-C motif) ligand 2, interleukin-23, long non-coding ribonucleic acid, messenger ribonucleic acid, osteoclasts.
\end{abstract}

Interleukin (IL)-23 is a heterodimeric cytokine composed of a p19 subunit and an IL-12 p40 subunit, which is mainly produced by activated macrophages and dendritic cells. ${ }^{1}$ Accompanying with IL-17, IL-23 displays proinflammatory properties ${ }^{2-4}$ and plays an important role in autoimmunity and chronic inflammation, which formed the IL-23/IL-17 axis. ${ }^{5}$ Previous studies showed that IL-23 was involved in the development of inflammatory arthritis such as rheumatoid arthritis (RA), psoriatic arthritis (PsA) and ankylosing spondylitis (AS). The concentration of IL-23 is elevated in serum and synovial fluid of patients with RA, which was positively associated with the disease activity and severity. ${ }^{6-8}$ In addition, the variants of single nucleotide polymorphisms in the genes encoding IL-23 receptor and IL-23 were also associated with susceptibility to spondyloarthritis like PsA or AS. ${ }^{9-12}$ Furthermore, IL-23 antagonist ustekinumab has excellent efficacy in PsA. It is well known that osteoclasts (OCs) are in charge of bone resorption while osteoblasts

Received: March 21, 2019 Accepted: May 13, 2019 Published online: April 27, 2020

Correspondence: Sheng-Ming Dai, MD. Department of Rheumatology \& Immunology, Shanghai Jiao Tong University Affiliated Sixth People's Hospital, 200233 Shanghai, China. Tel: 86-21-24056467 e-mail: shengmingdai@163.com

Chen M, Pang DD, Dai SM. Expression Profile of Osteoclasts following the Stimulation With Interleukin-23 in Mice. Arch Rheumatol $2020 ; 35(4): 533-544$. 
(OBs) are responsible for bone formation. The dynamic balance between $\mathrm{OC}$ and $\mathrm{OB}$ activities contributes to normal bone remodeling. Dysregulation of osteoclastogenesis that results in enhanced bone resorption is a characteristic feature of inflammatory arthritis. IL-23 may play an important role in osteoclastogenesis and maintenance of bone mass. ${ }^{13}$ Receptor activator of nuclear factor kappa B (RANK), which is expressed on $\mathrm{OC}$ precursors induces their differentiation into bone-resorbing mature OCs upon stimulation with RANK ligand (RANKL) and macrophagecolony stimulating factor (M-CSF). ${ }^{14-16}$ IL-23 has shown pro-osteoclastogenic capacity via both RANKL/RANK dependent and independent pathways, thereby aggravating joint damage and systemic bone loss. ${ }^{17-20}$ In addition, IL-23 can induce $\mathrm{OC}$ differentiation in other ways. The work of Shin et al. ${ }^{21}$ has shown that IL-23 acts on human $\mathrm{CD}_{16}{ }^{+} /$myeloid DNAX activating protein of $12 \mathrm{kDa}$-associated lectin-1+/DNAX activating protein of $12 \mathrm{kDa}^{+} \mathrm{OC}$ precursor population to induce OC differentiation. Leukotriene B4 and its receptor BLT1 also have critical roles in IL-23 induced myelopoiesis and osteoclastogenesis. ${ }^{22}$ However, the underlying mechanism where IL-23 modulates the function of OCs in inflammatory arthritis is still unknown. Therefore, in this study, we aimed to analyze the expression profile of OCs following the stimulation with IL-23 in mice, which would imply the underlying effects of IL-23 on the function of OCs in inflammatory arthritis.

\section{MATERIALS AND METHODS}

This study was conducted at Shanghai Jiao Tong University Affiliated Sixth People's Hospital between January 2017 and January 2018. Bone marrow monocytes were obtained from 5 male C57BL/6 mice (age 6 weeks; weighing $18-20 \mathrm{~g})$ and cultured in the presence of M-CSF (50 ng/mL) for four days, and then the cultures were further stimulated with soluble receptor activator of RANKL (30 ng/mL) in the presence of M-CSF (50 ng/mL) for six days. The cells were fixed and stained for tartrate-resistant acid phosphatase (TRAP), using a commercial kit. All the multinucleated (more than or equal to three nuclei) TRAP positive cells were mature OCs. In the following experiments, the mature OCs were cultured in the presence of IL-23 $(30 \mathrm{ng} / \mathrm{mL})$ or vehicle for four hours. The flow chart of the study was shown in Figure 1. All animal experiments were approved by the Institutional Animal Care and Use Committee of the Shanghai Jiao Tong University Affiliated Sixth People's Hospital.

The Agilent SurePrint G3 Mouse GE V2.0 Microarray (Agilent Technologies, Design ID: 074809, Agilent Technologies Inc., Santa Clara, CA, USA) was used to analyze the gene expression profiles of the six samples in this study (three samples from each group). Each glass slide was formatted with eight high-definition 60K array and screened for 56605 genes, most of which were messenger ribonucleic acids (mRNAs), and a few long non-coding RNAs (lncRNAs) as well. Total RNAs were quantified by the NanoDrop ND-2000 (Thermo Fisher Scientific, Wilmington, DE, USA)

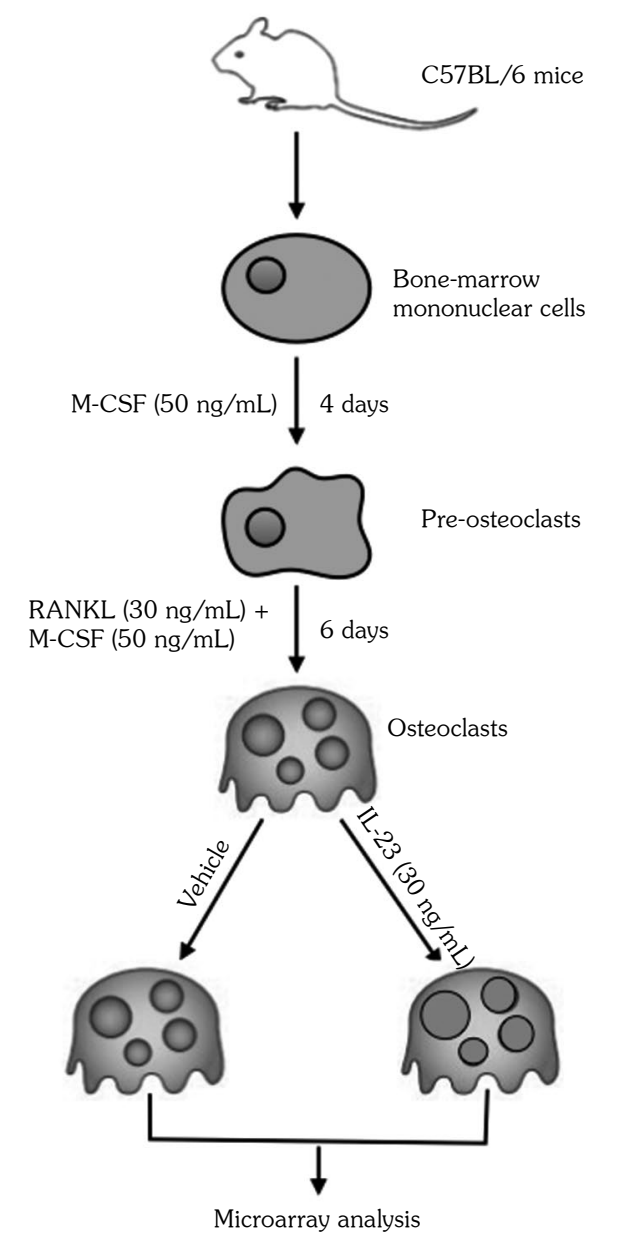

Figure 1. Flowchart of study.

M-CSF: Macrophage-colony stimulating factor; RANKL: Receptor activator of nuclear factor kappa B ligand; IL-23: Interleukin-23. 
and the RNAs integrity was assessed using Agilent Bioanalyzer 2100 (Agilent Technologies Inc., Santa Clara, CA, USA). Based on the manufacturer's standard protocols, the sample labeling, microarray hybridization and washing were performed. In brief, total RNAs were transcribed to double stranded complementary deoxyribonucleic acids, then synthesized into cRNAs and labeled with cyanine 3 labeled analog of cytidine triphosphate (3-CTP). The labeled cRNAs were hybridized onto the microarray. After washing, the arrays were scanned by the Agilent Scanner G2505C (Agilent Technologies Inc., Santa Clara, CA, USA).

The volcano plot was applied to screen out the genes based on their expression levels between the two groups. After analyzing the differentially expressed genes between the two groups by t-test, the volcano plot was drawn according to statistical significance [-log10 ( $p$ value) as $y$-axis] and the magnitude of change [log2 (fold change) as x-axis]. The hierarchical cluster analysis was performed to identify distinguishable mRNA expression profiles among all the samples. The differentially expressed mRNAs that have assigned gene symbol between the samples were shown as a heat map under hierarchical cluster analysis.

Gene ontology (GO) analysis was performed to explore the function of differentially expressed genes (http://www.geneontology.org). It contains three parts, which are biological process, cellular component and molecular function. The number of differential genes included in each GO category was calculated, and the two-sided Fisher's exact test was performed to classify the GO category and the GO annotation list. The small $p$ value indicated that the gene was enriched in this GO annotation. The cut-off of $p$ value was set as 0.05 to select genes for subsequent studies. Kyoto Encyclopedia of Genes and Genomes was used to perform the pathway analysis. The enrichment was calculated in a similar manner as the GO analysis.

The expression levels of some differentially expressed mRNAs were verified with quantitative real-time polymerase chain reaction (qPCR). Total RNAs were extracted using TRIzol (Thermo Fisher Scientific Inc., Waltham, MA, USA) and then reversely transcribed. The expression level of mRNA was represented as fold change using $2^{-\Delta \Delta \mathrm{Ct}}$ methods. The expression differences of mRNAs between two groups were analyzed by Student's t-test. Glyceraldehyde-3-phosphate dehydrogenase was used as an internal control to standardize mRNA expression level. The primer sequences used in this study were shown in Supplementary Table 1.

\section{Statistical analysis}

Feature Extraction software version 10.7.1.1 (Agilent Technologies Inc., Santa Clara, CA, USA) was used to analyze array images to get raw data. GeneSpring version 13.1 (Agilent Technologies Inc., Santa Clara, CA, USA) was employed to perform the basic analysis of the raw data. Firstly, the raw data were normalized with the quantile
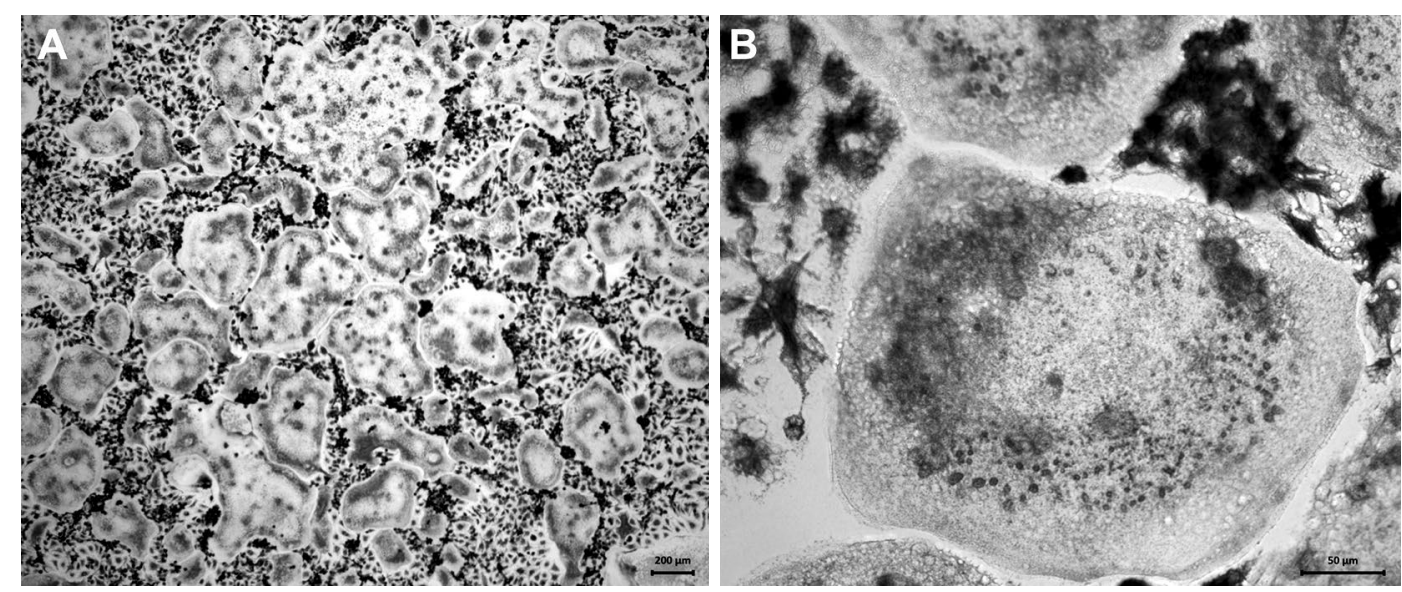

Figure 2. Osteoclast formation induced from mouse bone marrow monocytes. Tartrate-resistant acid phosphatase staining to identify mature osteoclasts. Scale bars, $200 \mu \mathrm{m}$ in panel A and $50 \mu \mathrm{m}$ in panel B. 
algorithm. The probes with at least $100 \%$ of the values in anyone out of all conditions that had flags in "detected" were chosen for further data analysis. Differentially expressed genes were then identified through fold change as well as $p$ value calculated with t-test. The threshold set for up- and down-regulated genes was a fold change $\geq 1.5$ and $p$ value $<0.05$.

\begin{tabular}{|c|c|c|c|}
\hline Gene symbol & Fold change & $\mathrm{P}$ value & Changes \\
\hline Sf1 & 1.504 & 0.0067 & DR \\
\hline LOC105247398 & 1.504 & 0.0155 & DR \\
\hline Magel2 & 1.513 & 0.0065 & DR \\
\hline Kazn & 1.524 & 0.0309 & DR \\
\hline Catsper4 & 1.525 & 0.0285 & DR \\
\hline Lrrc2 & 1.586 & 0.0081 & DR \\
\hline 2310005E17Rik & 1.603 & 0.0273 & DR \\
\hline Snhg7os & 1.618 & 0.0497 & DR \\
\hline 8030411F24Rik & 1.644 & 0.0485 & DR \\
\hline Gm15401 & 1.666 & 0.0112 & DR \\
\hline Sbp & 1.687 & 0.0172 & DR \\
\hline Vmn1r217 & 1.750 & 0.0050 & DR \\
\hline 4930517J16Rik & 1.772 & 0.0135 & DR \\
\hline Gm10866 & 1.773 & 0.0114 & DR \\
\hline Kcnj4 & 1.905 & 0.0105 & DR \\
\hline Gm28382 & 1.955 & 0.0493 & DR \\
\hline Gm12128 & 1.978 & 0.0426 & DR \\
\hline Yipf6 & 2.020 & 0.0312 & DR \\
\hline Plek2 & 2.058 & 0.0020 & DR \\
\hline Samd4 & 2.720 & 0.0169 & DR \\
\hline Arhgap28 & 1.509 & 0.0132 & UR \\
\hline Nfasc & 1.516 & 0.0427 & UR \\
\hline Cxcl1 & 1.526 & 0.0121 & UR \\
\hline Shisa9 & 1.536 & 0.0013 & UR \\
\hline Trappc12 & 1.540 & 0.0317 & UR \\
\hline Fcrlb & 1.541 & 0.0447 & UR \\
\hline Atat1 & 1.576 & 0.0253 & UR \\
\hline $\mathrm{Cxcl} 2$ & 1.599 & 0.0138 & UR \\
\hline Kifc1 & 1.617 & 0.0124 & UR \\
\hline Ndrg2 & 1.637 & 0.0388 & UR \\
\hline H1f0 & 1.650 & 0.0496 & UR \\
\hline 2210018M11Rik & 1.654 & 0.0141 & UR \\
\hline Spag1 & 1.663 & 0.0125 & UR \\
\hline Tcap & 1.678 & 0.0288 & UR \\
\hline Dpf2 & 1.678 & 0.0093 & UR \\
\hline Arid $4 b$ & 1.759 & 0.0127 & UR \\
\hline Olfr699 & 1.946 & 0.0334 & UR \\
\hline
\end{tabular}




\section{RESULTS}

After the mouse bone marrow monocytes were cultured in the presence of M-CSF and RANKL, TRAP staining was used to identify the mature OCs. Mature OCs were successfully induced (Figure 2), and then stimulated with IL-23 or vehicle.

Based on the criteria of fold change $\geq 1.5$ as well as $p$ value $<0.05$, there were 55 mRNAs that were differentially expressed between the two groups. Among them, 23 genes were up-regulated and 32 genes were down-regulated, which were shown by volcano plot (Figure 3). Among these differentially expressed mRNAs, there were 37 mRNAs that had assigned gene symbols (Table 1). The relative expression levels of these 37 mRNAs were shown in a heat map with hierarchical clustering analysis (Figure 4).

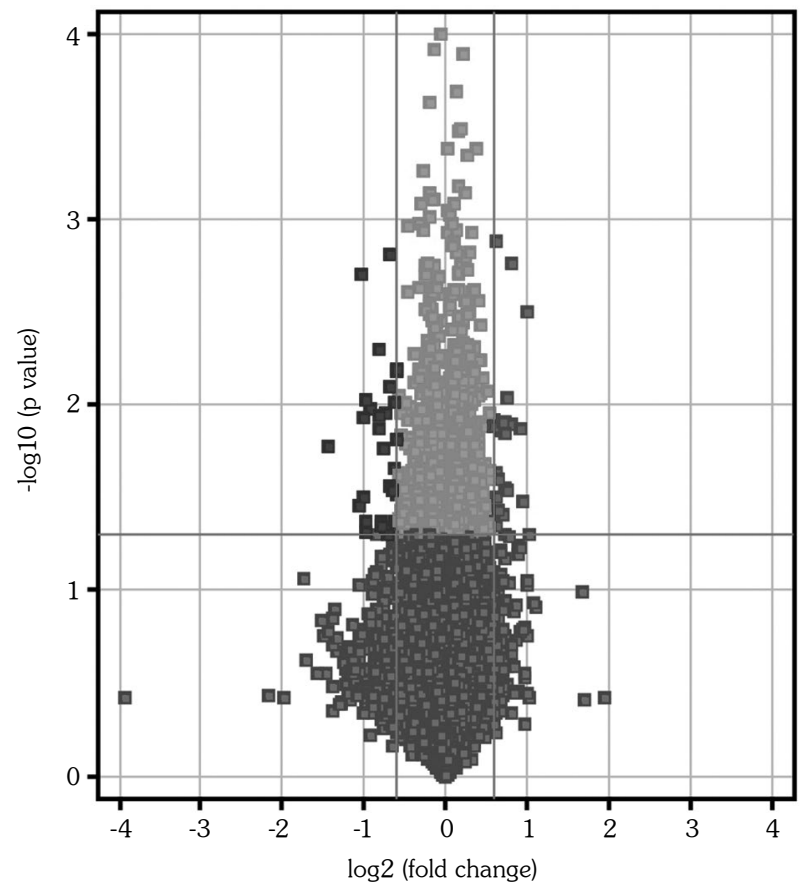

Figure 3. Volcano plot showing differentially expressed messenger ribonucleic acids. Vertical green lines correspond to a 1.5 fold up- and down-regulation while horizontal green line represents a $p$ value of 0.05 . The red dots and blue dots respectively represent the up-regulated and down-regulated messenger ribonucleic acids with more than 1.5 fold change and statistical significance. Statistical significance was defined as fold change $\geq 1.5$ and $p$ value $<0.05$ between two groups.
Gene ontology and pathway analyses was performed to explore the potential function of the 37 differentially expressed mRNAs. The most significantly enriched GO annotations in the classification of biological process, cellular component and molecular function among the differentially expressed mRNAs were respectively shown in Figure 5a-c. Accordingly, the differentially expressed mRNAs were related to cell chemotaxis, chemokine-mediated signaling pathway, cell junction and $\mathrm{C}-\mathrm{X}-\mathrm{C}$ chemokine receptors (CXCR). The pathway analysis showed that the IL-23-regulated mRNAs were related to tumor necrosis factor (TNF) signaling pathway, chemokine signaling pathway, cytokine-cytokine receptor interaction and nuclear factor (NF)-kappa B signaling pathway (Figure 6). The network of the differentially enriched genes was shown in Figure 7.

Considering the observed fold changes, the calculated $p$ values, and the gene function, we selected the following mRNAs to validate the array results: N-myc downstream-regulated gene 2 (Ndrg2), sterile alpha motif domain containing protein 4 (Samd4), chemokine

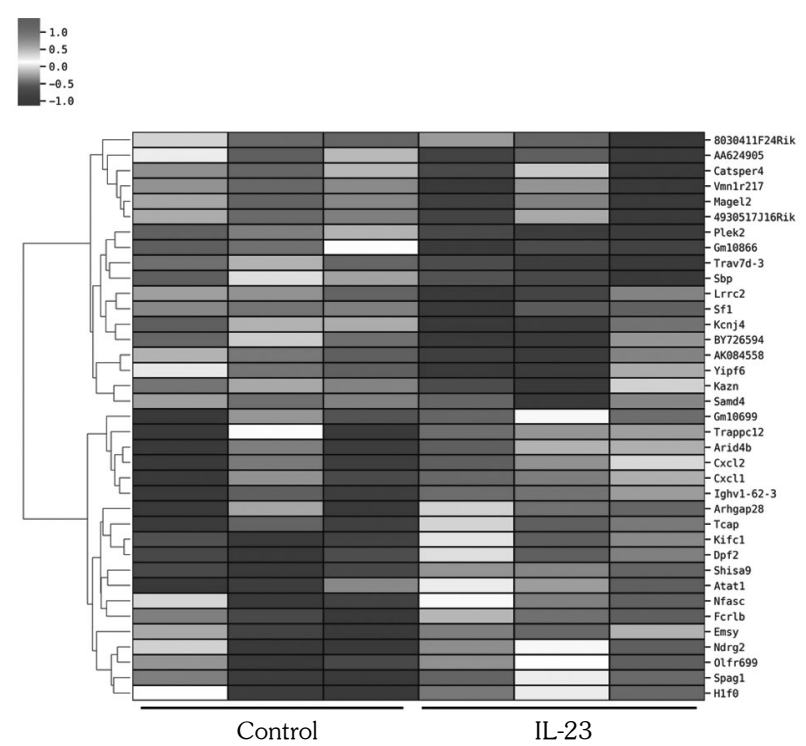

Figure 4. Heat map under hierarchical cluster analysis of differentially expressed messenger ribonucleic acids. Hierarchical cluster showed that differentially expressed genes ultimately clustered into two major branches, including up-regulated genes, which were shown in red, and down-regulated genes, which were shown in blue. Color depth represents relative gene expression levels. IL-23: Interleukin-23. 
(a)

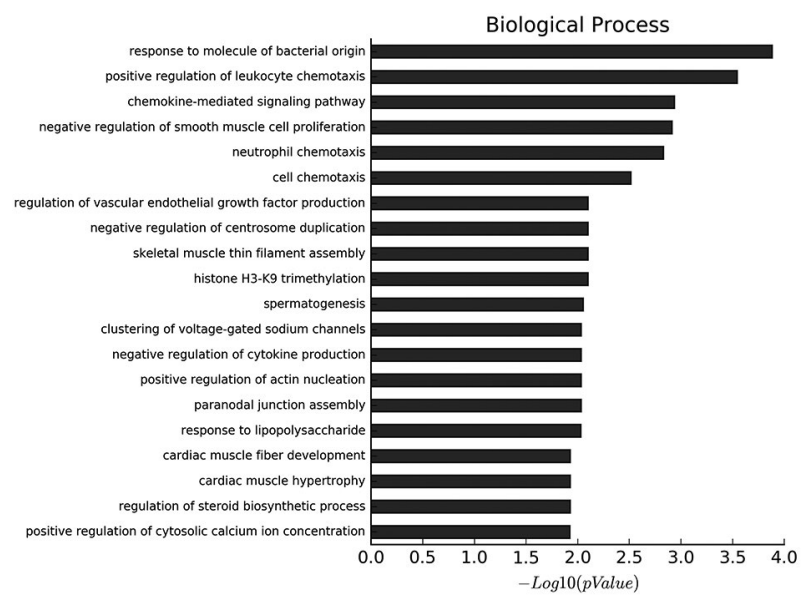

(b)

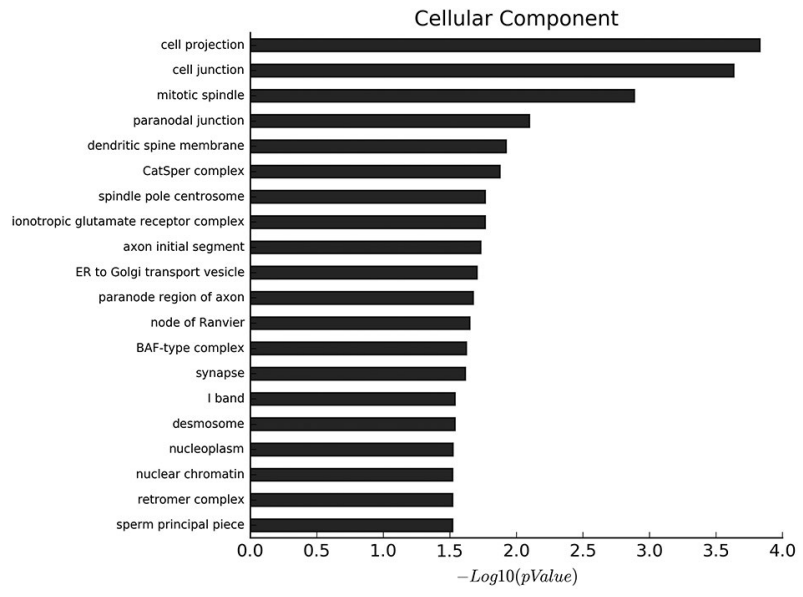

(c)

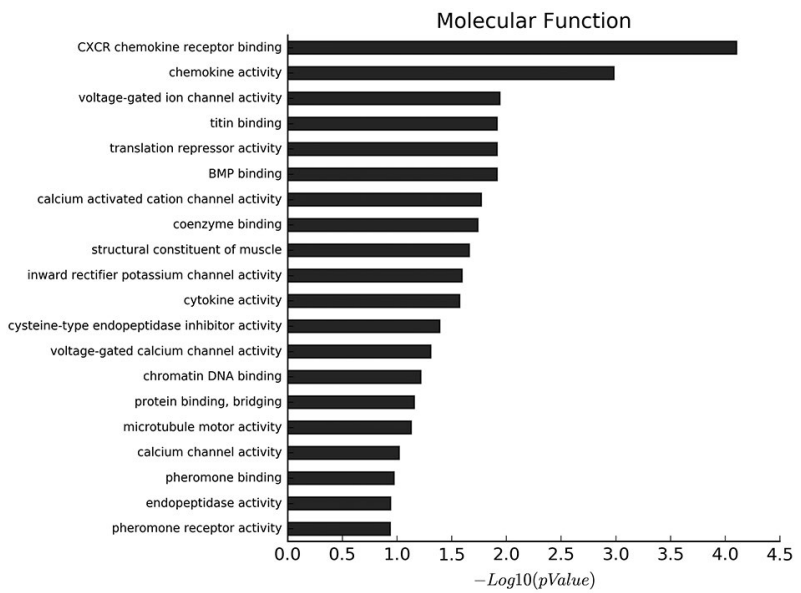

Figure 5. Most significantly enriched gene ontology annotations among differentially expressed messenger ribonucleic acids. (a) Top-ten significant changes in gene ontology biological process classification. (b) Top-ten significant changes in gene ontology cellular component classification. (c) Top-ten significant changes in gene ontology molecular function classification.
(C-X-C motif) ligand 1 (Cxcl1) and chemokine (C-X-C motif) ligand 2 ( $\mathrm{Cxcl} 2)$. The expression trends of all these genes were consistent with microarray data. However, the differences of Ndrg2 and Samd4 did not reach statistical significance ( $p>0.05)$. The expression levels of Cxcl1 and Cxcl2 were significantly up-regulated under the stimulation of IL-23 (Figure 8).

There were 18 differentially expressed lncRNAs between the two groups, which consisted of four up-regulated lncRNAs and 14 down-regulated lncRNAs according to the criteria of fold change

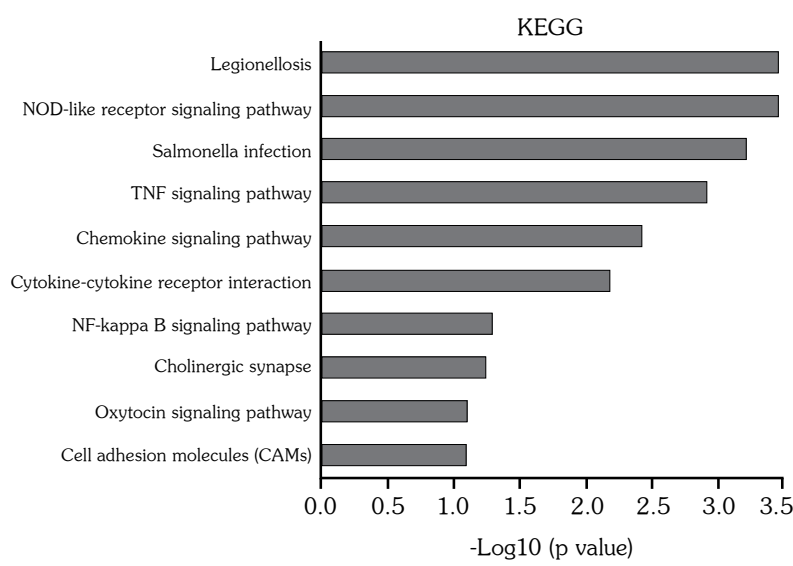

Figure 6. Top-ten significantly enriched pathways among differentially expressed messenger ribonucleic acids.

KEGG: Kyoto Encyclopedia of Genes and Genomes; NOD: Nucleotidebinding oligomerization domain; TNF: Tumor necrosis factor; NF: Nuclear factor

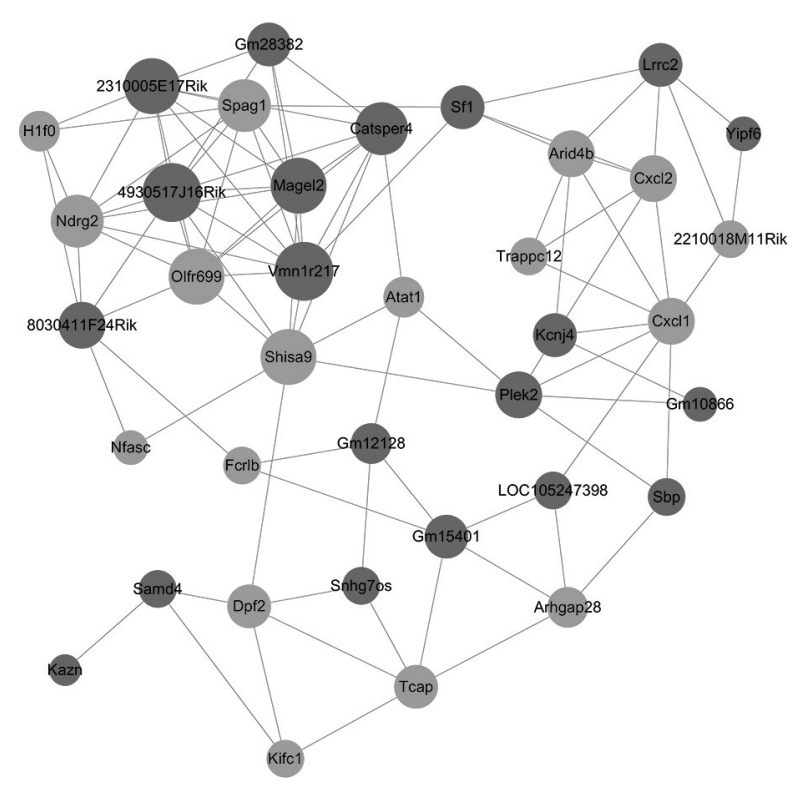

Figure 7. Network of differentially enriched genes. 

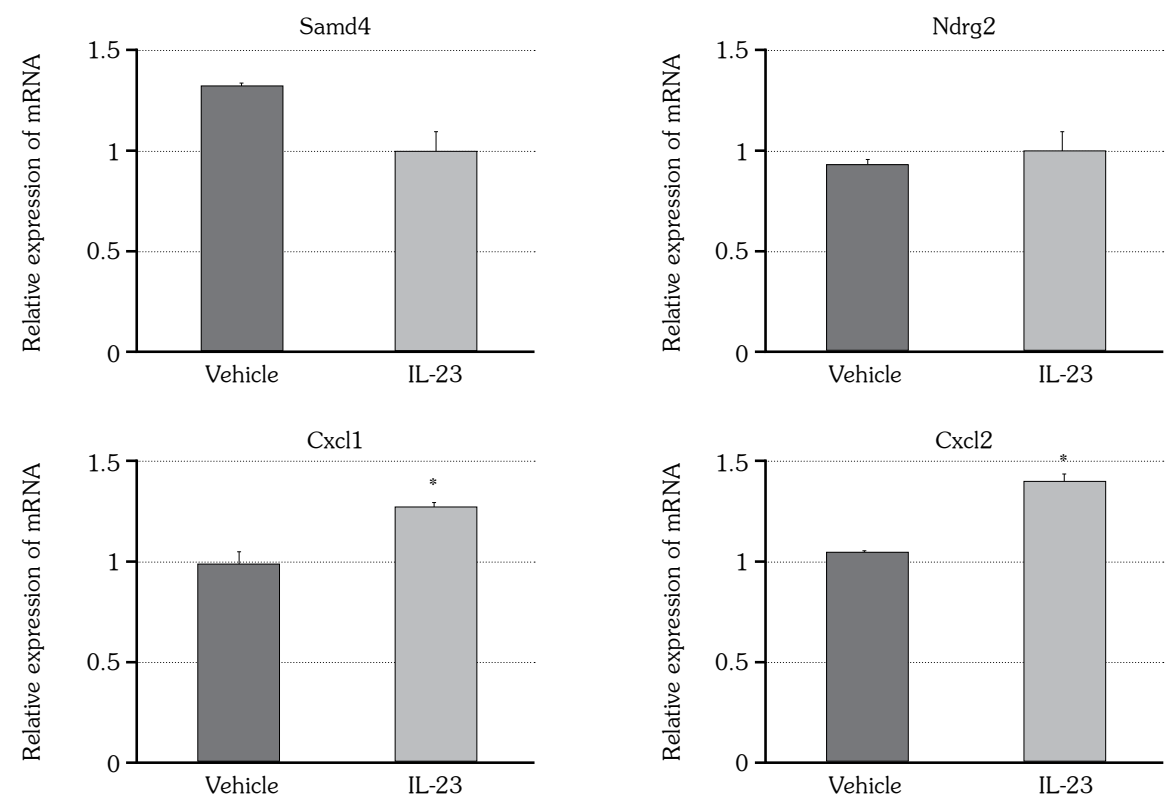

Figure 8. Validation of differentially expressed messenger ribonucleic acids using real-time polymerase chain reaction. Glyceraldehyde-3-phosphate dehydrogenase was used as an internal control to standardize messenger ribonucleic acid expression level. Relative expression levels of sterile alpha motif domain containing protein 4, N-myc downstream-regulated gene 2, chemokine (C-X-C motif) ligand 1 and chemokine ( $\mathrm{C}-\mathrm{X}-\mathrm{C}$ motif) ligand 2 were evaluated in osteoclasts following stimulation with interleukin $23(n=3)$ or vehicle $(n=3)$. Values are mean \pm standard error of mean. * $p$ value $<0.05$; mRNA: Messenger ribonucleic acid; Samd4: Sterile alpha motif domain containing protein 4; IL-23: Interleukin-23; Ndrg2: N-myc downstream-regulated gene 2; Cxcl1: Chemokine (C-X-C motif) ligand 1; $\mathrm{Cxcl} 2$ : Chemokine (C-X-C motif) ligand 2.

$\geq 1.5$ as well as $p$ value $<0.05$ (Table 2). The hierarchical clustering analysis was also applied, as shown in the Figure 9. The co-expression networks were constructed with all of the differentially expressed lncRNAs and mRNAs in this study. The lncRNAs and mRNAs that had Pearson correlation coefficients $\geq 0.7$ and $p$ value $<0.05$ were selected, and subsequently a network was constructed (Figure 10).

\section{DISCUSSION}

Although the role of IL-23 in OC formation has been investigated in some researches, the potential effects of IL-23 on the function of OCs and the mechanism of it involved in the pathogenesis of inflammatory arthritis are still unknown. In an attempt to elucidate these issues, we performed a preliminary study to analyze the impact of IL-23 on the gene expression profile in
OCs. As a result, we found that expression levels of more than 37 genes in OCs were modulated by IL-23, implying that IL-23 may also modulate the function of mature OCs.

Functional group analyses were performed to reveal the biological function of the genes regulated by IL-23. According to GO analysis, the IL-23 regulated genes are mainly involved in chemokine-mediated signaling pathway, cell chemotaxis, CXCR chemokine receptor binding and chemokine activity. Consistent with this result, the pathway analysis identified that the IL-23 regulated genes were mainly associated with the inflammatory signaling pathways like TNF, chemokine, NF-kappa B and cytokine receptor interaction. In all these above biological functions and signaling pathways, the most enriched genes were Cxcl1 and Cxcl2. Their expression levels were also confirmed by the following $\mathrm{qPCR}$ validation. 


\begin{tabular}{|lccc|}
\hline \multicolumn{3}{l}{ Table 2. Differentially expressed lncRNAs in osteoclasts } & \multicolumn{3}{l}{ following the stimulation with } \\
Interleukin-23 & Fold change & P value & Changes \\
\hline Gene symbol & 1.666 & 0.0112 & DR \\
\hline Gm15401 & 1.504 & 0.0155 & DR \\
LOC105247398 & 1.543 & 0.0427 & DR \\
lincRNA:chr8:85952719-85958369 & 1.547 & 0.0219 & DR \\
lincRNA:chr16:30200995-30205965 & 1.603 & 0.0273 & DR \\
2310005E17Rik & 1.618 & 0.0497 & DR \\
Snhg7os & 1.654 & 0.0464 & DR \\
lincRNA:chr11:22500336-22520995 & 1.712 & 0.0451 & DR \\
lincRNA:chr5:113594167-113645342 & 1.713 & 0.0431 & DR \\
lincRNA:chr13:98513868-98517366 & 1.955 & 0.0493 & DR \\
Gm28382 & 1.977 & 0.0095 & DR \\
lincRNA:chr7:108253956-108254385 & 1.978 & 0.0426 & DR \\
Gm12128 & 2.018 & 0.0119 & DR \\
lincRNA:chr6:90436386-90469399 & 2.099 & 0.0351 & DR \\
lincRNA:chr6:91928958-91936887 & 1.544 & 0.0232 & UR \\
lincRNA:chr17:26911032-26912366 & 1.582 & 0.0367 & UR \\
lincRNA:chr13:99870660-99877535 & 1.891 & 0.0134 & UR \\
lincRNA:chr10:117021051-117038683 & 1.995 & 0.0032 & \\
lincRNA:chr17:34018371-34019394 & & & \\
\hline IncRNAs: Long non-coding ribonucleic acids; DR: Down-regulated; UR: Up-regulated. & \\
\hline
\end{tabular}

Chemokine ( $\mathrm{C}-\mathrm{X}-\mathrm{C}$ motif) ligand 1 and $\mathrm{Cxcl} 2$ belong to CXC chemokine family with 78\% homology of their sequence. ${ }^{23}$ They were first

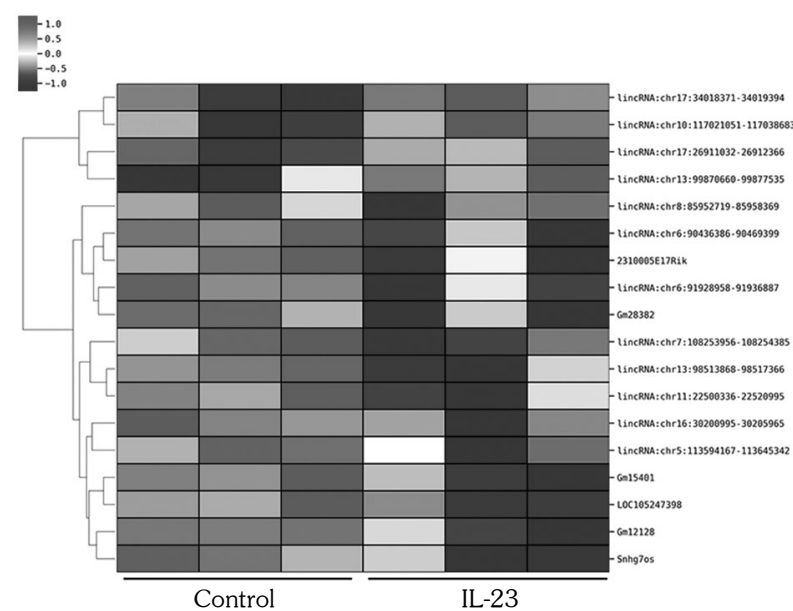

Figure 9. Heat map under hierarchical cluster analysis of differentially expressed IncRNAs. Hierarchical cluster showed that differentially expressed genes ultimately clustered into two major branches, including up-regulated genes, which were shown in red, and down-regulated genes, which were shown in blue. Color depth represents relative gene expression levels.

IncRNA: Long non-coding ribonucleic acid; IL-23: Interleukin-23. identified as major chemokines produced by endotoxin-treated macrophages and they function as inducers of inflammation. ${ }^{24}$ Both act through binding to the receptor CXCR2, a $\mathrm{G}$ protein-coupled receptor, ${ }^{25}$ to induce cellular adherence or cell-cell fusion. ${ }^{26}$ In addition to functioning as chemoattractants during inflammation and potentially as inducers of pre-OC fusion, they were also identified as necessary for $\mathrm{OC}$ formation. According to the study of Hardaway et al. ${ }^{27} \mathrm{Cxcl} 1$ and $\mathrm{Cxcl} 2$ chemokines stimulate OCs differentiation in vitro. Treatment with either recombinant $\mathrm{Cxcl} 1$ or $\mathrm{Cxcl} 2$ enhanced OC maturation compared to control conditions. Similar to the present study, Ha et al. ${ }^{28}$ also found that the expression of $\mathrm{Cxcl} 2$ in $\mathrm{OC}$ precursors could be enhanced by RANKL, and it has critical roles in osteoclastogenesis in vitro and in bone erosion in vivo. In addition, $\mathrm{Cxcl} 1$ and $\mathrm{Cxcl} 2$ also participate in lipopolysaccharide (LPS)-induced OC formation. It was found that they were significantly up-regulated in response to LPS and blockade of $\mathrm{Cxcl} 1$ and $\mathrm{Cxcl} 2$ nearly ablated OC formation induced by LPS stimulation. ${ }^{29}$ All these data suggest that $\mathrm{Cxcl} 1$ and $\mathrm{Cxcl} 2$ not only function as chemoattractants 


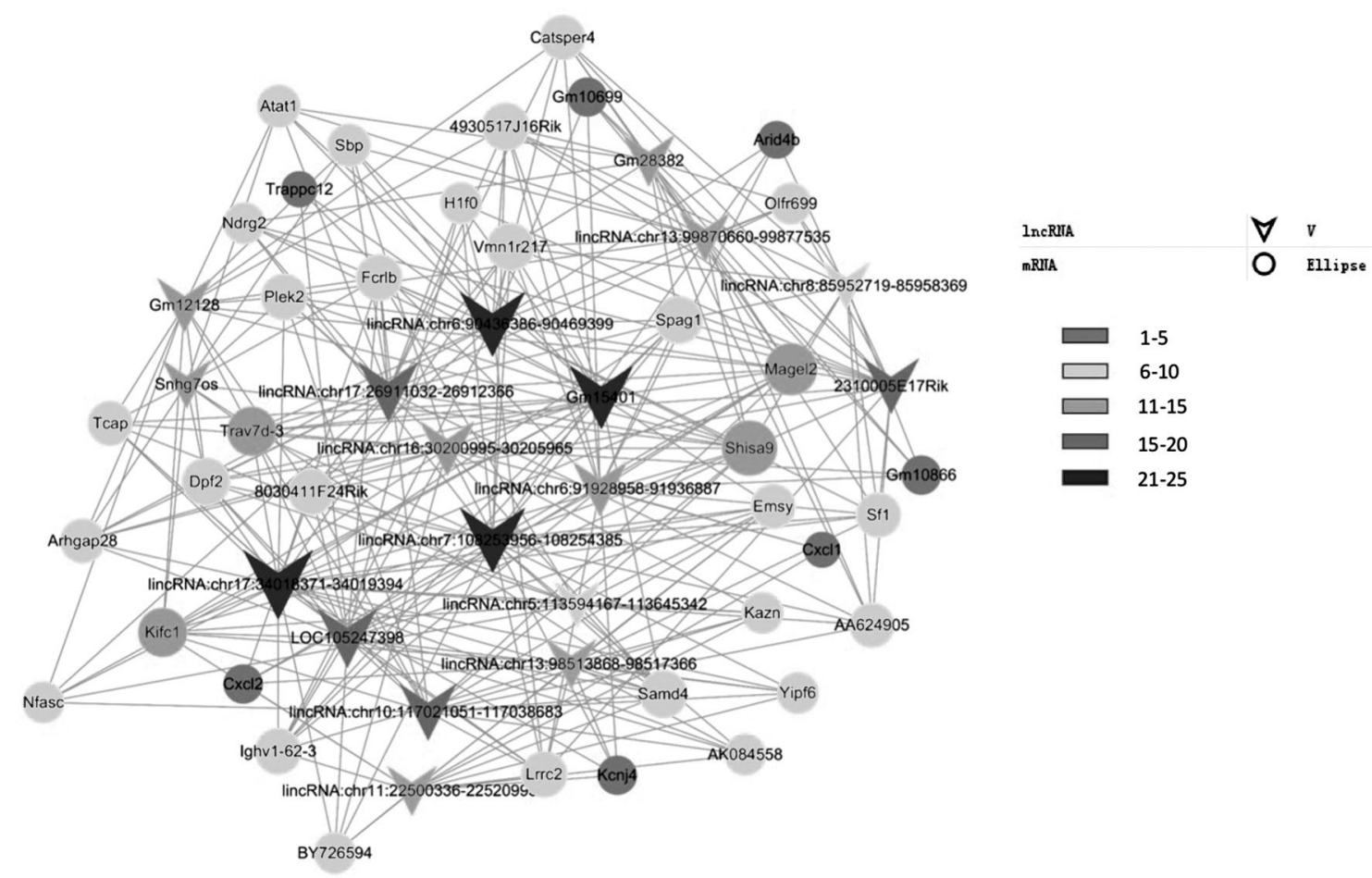

Figure 10. Co-expression networks of all differentially expressed long non-coding ribonucleic acids and messenger ribonucleic acids. A circle represents a messenger ribonucleic acid and an inverted triangle represents a long non-coding ribonucleic acid. Line between them represents significant co-expression of them. K-core was used to show number of co-expression genes associated with this gene. Different colors represent different ranges of k-core. Size of circle or triangle also represents number of co-expression genes associated with this gene.

IncRNA: Long non-coding ribonucleic acid; mRNA: Messenger ribonucleic acid.

for leukocytes during inflammation but also play important roles in OC formation. In our study, the expression of $\mathrm{Cxcl} 1$ and $\mathrm{Cxcl} 2$ in OCs were significantly up-regulated by IL-23. This result suggests that through modulating the expression of Cxcl1 and $\mathrm{Cxcl} 2$ in OCs, IL-23 might enhance the leukocyte chemotaxis as well as OC formation, thus promote the inflammatory and bone resorption in the pathogenesis of inflammatory arthritis.

Considering the statistical significance as well as the gene function, other two genes including Ndrg2 and Samd4 were also selected to validate the array results. Consequently, the expression level trends of these genes were consistent with microarray data, whereas the differences did not reach statistical significance. Ndrg2 belongs to the NDRG family, which is a family of differentiation-related genes including the proteins Ndrg1-4. Highly expressed in the tissue of brain, salivary glands, and skeletal muscle, Ndrg2 has been identified as a regulator of dendritic cell differentiation from monocytes. ${ }^{30}$ Besides the role of regulation on cell growth, apoptosis, and neurodegeneration, ${ }^{31-33}$ it also plays an important role in modulating the OC differentiation. ${ }^{34}$ Kang et $\mathrm{al}^{34}$ showed that NDRG2 overexpression inhibited the differentiation of U937 cells into OCs in response to stimulation of M-CSF and RANKL. In the present study, the expression of Ndrg2 in OCs was up-regulated by the stimulation of IL-23. According to the role of Ndrg2 on OC differentiation previously reported, we speculate that increased expression of $\mathrm{Ndrg} 2$ is a kind of negative feedback to the increase of $\mathrm{OC}$ differentiation under the stimulation of IL-23.

Sterile alpha motif domain containing protein 4 is a translational repressor which works by directly binding to RNA. It has been identified as a regulator for skeletogenesis by down-regulation of $\mathrm{OB}$ and chondrocyte differentiation. ${ }^{35}$ To our knowledge, the effects of Samd4 on the OC 
differentiation have not been revealed in previous studies. Here, we identified that the expression of Samd4 was decreased in OCs stimulated with IL-23. This result suggests that Samd 4 might play a role in OC differentiation and participate in the development of disease.

The lncRNAs expression profile of OCs following the stimulation with IL-23 was analyzed at the same time. Recently, the biological function of non-coding RNAs has attracted more attention. With the development of sequencing technology and microarray, a large number of non-coding RNAs including lncRNAs have been identified. Increasing evidence revealed that lncRNAs might have interaction with mRNA or microRNA, thus playing important roles in regulation of genes expression. Several lncRNAs have been identified to contribute to inflammatory arthritis such as RA. ${ }^{36}$ In this study, we identified that lncRNAs of OCs were regulated following the stimulation with IL-23, and some of them had co-expression with differentially expressed mRNAs. These results revealed that lncRNAs of OCs were also regulated by IL-23, suggesting that they may play a role in the pathogenesis of inflammatory arthritis. However, due to the limited number of lncRNAs that can be screened by the microarray used in this study, the functions of these differentially expressed lncRNAs have not been studied further. The role of lncRNAs in inflammatory arthritis and underlying mechanism needs to be confirmed by further studies.

The current study revealed that IL-23 regulated the gene expression profile including mRNAs and lncRNAs in OCs, thus IL-23 might modulate the function of OCs in inflammatory arthritis. Deciphering the precise molecular mechanisms of these coding and non-coding RNAs function in inflammatory arthritis would be vital to provide insight into the pathogenesis of disease and to explore new potential targets for therapy.

There are several limitations of this study that should be noted. The main limitation was that the functional experiments were not performed to validate the results. Functional experiments will be carried out in the future to elucidate the potential mechanism. In addition to this is the relatively small sample size. Thus these data may be confirmed in larger-scale studies. Lastly, the expression levels of differentially expressed
lncRNAs were not verified. Due to the limited IncRNA data contained in the current microarray, the differentially expressed lncRNAs were not further analyzed. The lncRNAs expression profile of OCs following the stimulation with IL-23 and their interaction with mRNAs can be explored in future studies.

In conclusion, we revealed the expression profile of OCs including mRNAs and lncRNAs following the stimulation of IL-23 in mice. The expression of Cxcl1 and $\mathrm{Cxcl} 2$ in OCs were significantly regulated by IL-23, suggesting that IL-23 might promote the inflammatory and bone resorption in the pathogenesis of inflammatory arthritis through enhancing the leukocyte chemotaxis as well as OC formation. Several lncRNAs of OCs were also regulated by IL-23, which needs to be confirmed by further studies. Although these results are preliminary findings at this stage, they might be valuable in providing targets for further research on the disease pathogenesis.

\section{Declaration of conflicting interests}

The authors declared no conflicts of interest with respect to the authorship and/or publication of this article.

\section{Funding}

This project was supported by a grant from National Natural Science Foundation of China (No. 81471604) and a grant from National Key Basic Research Program of China (2014CB541804).

\section{REFERENCES}

1. Duvallet E, Semerano L, Assier E, Falgarone G, Boissier MC. Interleukin-23: a key cytokine in inflammatory diseases. Ann Med 2011;43:503-11.

2. Burmester GR, Feist E, Dörner T. Emerging cell and cytokine targets in rheumatoid arthritis. Nat Rev Rheumatol 2014;10:77-88.

3. Stamp LK, Easson A, Pettersson L, Highton J, Hessian PA. Monocyte derived interleukin (IL)-23 is an important determinant of synovial IL-17A expression in rheumatoid arthritis. $\mathrm{J}$ Rheumatol 2009;36:2403-8.

4. Chang J, Voorhees TJ, Liu Y, Zhao Y, Chang $\mathrm{CH}$. Interleukin-23 production in dendritic cells is negatively regulated by protein phosphatase 2A. Proc Natl Acad Sci U S A 2010;107:8340-5.

5. Gaffen SL, Jain R, Garg AV, Cua DJ. The IL-23-IL-17 immune axis: from mechanisms to therapeutic testing. Nat Rev Immunol 2014;14:585-600. 
6. Abu Al Fadl EM, Fattouh M, Allam AA. High IL-23 level is a marker of disease activity in rheumatoid arthritis. Egypt J Immunol 2013;20:85-92.

7. Guo YY, Wang NZ, Zhao S, Hou LX, Xu YB, Zhang N. Increased interleukin-23 is associated with increased disease activity in patients with rheumatoid arthritis. Chin Med J (Engl) 2013;126:850-4.

8. Metawi SA, Abbas D, Kamal MM, Ibrahim MK. Serum and synovial fluid levels of interleukin-17 in correlation with disease activity in patients with RA. Clin Rheumatol 2011;30:1201-7.

9. Rueda B, Orozco G, Raya E, Fernandez-Sueiro $\mathrm{JL}$, Mulero J, Blanco FJ, et al. The IL23R Arg381Gln non-synonymous polymorphism confers susceptibility to ankylosing spondylitis. Ann Rheum Dis 2008;67:1451-4.

10. Coffre M, Roumier M, Rybczynska M, Sechet E, Law HK, Gossec L, et al. Combinatorial control of Th17 and Th1 cell functions by genetic variations in genes associated with the interleukin-23 signaling pathway in spondyloarthritis. Arthritis Rheum 2013;65:1510-21.

11. Bowes J, Orozco G, Flynn E, Ho P, Brier R, MarzoOrtega $\mathrm{H}$, et al. Confirmation of TNIP1 and IL23A as susceptibility loci for psoriatic arthritis. Ann Rheum Dis 2011;70:1641-4.

12. Filer C, Ho P, Smith RL, Griffiths C, Young HS, Worthington $\mathrm{J}$, et al. Investigation of association of the IL12B and IL23R genes with psoriatic arthritis. Arthritis Rheum 2008;58:3705-9.

13. Adamopoulos IE, Tessmer M, Chao CC, Adda S, Gorman D, Petro M, et al. IL-23 is critical for induction of arthritis, osteoclast formation, and maintenance of bone mass. J Immunol 2011;187:951-9.

14. Theill LE, Boyle WJ, Penninger JM. RANK-L and RANK: T cells, bone loss, and mammalian evolution. Annu Rev Immunol 2002;20:795-823.

15. Tanaka S, Takahashi N, Udagawa N, Tamura T, Akatsu $\mathrm{T}$, Stanley ER, et al. Macrophage colony-stimulating factor is indispensable for both proliferation and differentiation of osteoclast progenitors. J Clin Invest 1993;91:257-63.

16. Nakagawa N, Kinosaki M, Yamaguchi K, Shima $\mathrm{N}$, Yasuda $\mathrm{H}$, Yano $\mathrm{K}$, et al. RANK is the essential signaling receptor for osteoclast differentiation factor in osteoclastogenesis. Biochem Biophys Res Commun 1998;253:395-400.

17. Sato K, Suematsu A, Okamoto K, Yamaguchi A, Morishita Y, Kadono Y, et al. Th17 functions as an osteoclastogenic helper $\mathrm{T}$ cell subset that links $\mathrm{T}$ cell activation and bone destruction. $\mathrm{J}$ Exp Med 2006;203:2673-82.

18. Li X, Kim KW, Cho ML, Ju JH, Kang CM, Oh HJ, et al. IL-23 induces receptor activator of NF-kappaB ligand expression in fibroblast-like synoviocytes via STAT3 and NF-kappaB signal pathways. Immunol Lett 2010;127:100-7.

19. Chen L, Wei XQ, Evans B, Jiang W, Aeschlimann D. IL-23 promotes osteoclast formation by up-regulation of receptor activator of NF-kappaB (RANK) expression in myeloid precursor cells. Eur $\mathrm{J}$ Immunol 2008;38:2845-54.

20. Razawy W, van Driel M, Lubberts E. The role of IL-23 receptor signaling in inflammation-mediated erosive autoimmune arthritis and bone remodeling. Eur $\mathrm{J}$ Immunol 2018;48:220-9.

21. Shin HS, Sarin R, Dixit N, Wu J, Gershwin E, Bowman EP, et al. Crosstalk among IL-23 and DNAX activating protein of $12 \mathrm{kDa}$-dependent pathways promotes osteoclastogenesis. J Immunol 2015;194:316-24.

22. Bouchareychas L, Grössinger EM, Kang M, Qiu H, Adamopoulos IE. Critical Role of LTB4/BLT1 in IL-23Induced Synovial Inflammation and Osteoclastogenesis via NF-кB. J Immunol 2017;198:452-60.

23. Semple BD, Kossmann T, Morganti-Kossmann MC. Role of chemokines in CNS health and pathology: a focus on the CCL2/CCR2 and CXCL8/CXCR2 networks. J Cereb Blood Flow Metab 2010;30:459-73.

24. Wolpe SD, Cerami A. Macrophage inflammatory proteins 1 and 2: members of a novel superfamily of cytokines. FASEB J 1989;3:2565-73.

25. Veenstra M, Ransohoff RM. Chemokine receptor CXCR2: physiology regulator and neuroinflammation controller? J Neuroimmunol 2012;246:1-9.

26. Ha J, Lee Y, Kim HH. CXCL2 mediates lipopolysaccharide-induced osteoclastogenesis in RANKL-primed precursors. Cytokine 2011;55:48-55.

27. Hardaway AL, Herroon MK, Rajagurubandara E, Podgorski I. Marrow adipocyte-derived CXCL1 and CXCL2 contribute to osteolysis in metastatic prostate cancer. Clin Exp Metastasis 2015;32:353-68.

28. Ha J, Choi HS, Lee Y, Kwon HJ, Song YW, Kim HH. $\mathrm{CXC}$ chemokine ligand 2 induced by receptor activator of NF-kappa B ligand enhances osteoclastogenesis. J Immunol 2010;184:4717-24.

29. Valerio MS, Herbert BA, Basilakos DS, Browne C, Yu H, Kirkwood KL. Critical role of MKP-1 in lipopolysaccharide-induced osteoclast formation through CXCL1 and CXCL2. Cytokine 2015;71:71-80.

30. Choi SC, Kim KD, Kim JT, Kim JW, Yoon DY, Choe YK, et al. Expression and regulation of NDRG2 (N-myc downstream regulated gene 2) during the differentiation of dendritic cells. FEBS Lett 2003;553:413-8.

31. Deng Y, Yao L, Chau L, Ng SS, Peng Y, Liu X, et al. N-Myc downstream-regulated gene 2 (NDRG2) inhibits glioblastoma cell proliferation. Int $J$ Cancer 2003;106:342-7.

32. Mitchelmore C, Büchmann-Møller S, Rask L, West MJ, Troncoso JC, Jensen NA. NDRG2: a novel Alzheimer's disease associated protein. Neurobiol Dis 2004;16:48-58.

33. Takahashi K, Yamada M, Ohata H, Honda K, Yamada M. Ndrg2 promotes neurite outgrowth of NGF-differentiated PC12 cells. Neurosci Lett 2005;388:157-62. 
34. Kang K, Nam S, Kim B, Lim JH, Yang Y, Lee $\mathrm{MS}$, et al. Inhibition of osteoclast differentiation by overexpression of NDRG2 in monocytes. Biochem Biophys Res Commun 2015;468:611-6.

35. Niu N, Xiang JF, Yang Q, Wang L, Wei Z, Chen LL, et al. RNA-binding protein SAMD4 regulates skeleton development through translational inhibition of Mig6 expression. Cell Discov 2017;3:16050.

36. Zhang Y, Xu YZ, Sun N, Liu JH, Chen FF, Guan $\mathrm{XL}$, et al. Long noncoding RNA expression profile in fibroblast-like synoviocytes from patients with rheumatoid arthritis. Arthritis Res Ther 2016;18:227.

Supplementary Table 1. Primer sequence used in verification of differentially expressed mRNAs

\begin{tabular}{lll}
\hline Gene & Forward & Reverse \\
\hline Ndrg2 & 5'-ACACCTTATGGCTCGGTCAC-3' & 5'-TCTCTTGCATATCCCCGAAC-3' \\
Samd4 & 5'-GAACCCACGACAGTACCAGA-3' & 5'-TTTGTGGGGAAGTATGCACG-3' \\
Cxcl1 & 5'-CTGGGATTCACCTCAAGAACATC-3' & 5'-CAGGGTCAAGGCAAGCCTC-3' \\
Cxcl2 & 5'-CCAACCACCAGGCTACAGG-3' & 5'-GCGTCACACTCAAGCTCTG-3' \\
GAPDH & 5'-GCACAGTCAAGGCCGAGAAT-3' & 5'-GCCTTCTCCATGGTGGTGAA-3' \\
\hline
\end{tabular}

Ndrg2: N-Myc downstream regulated gene 2; Samd4: Sterile alpha motif domain containing protein 4; Cxcl1: Chemokine (C-X-C motif) ligand 1; Cxcl2: Chemokine (C-X-C motif) ligand 2; GAPDH: Glyceraldehyde phosphate dehydrogenase. 